\title{
Upgrades for TwinSol facility
}

\author{
P. D. O’Malley, D. W. Bardayan, J.J. Kolata, M. R. Hall, O. Hall, J. Allen \\ Physics Department, University of Notre Dame, Notre Dame, IN 46556, United States
}

F. D. Becchetti

Physics Department, U. Michigan, Ann Arbor MI 48109, United States

and the TwinSol collaboration

\begin{abstract}
TwinSol, a pair of coupled, superconducting solenoids, was one of the first devices capable of producing beams of radioactive nuclei at energies near the Coulomb barrier. A primary beam from University of Notre Dame (UND) tandem accelerator is used to bombard a primary target producing a secondary beam in flight. TwinSol is used to gather, separate, and focus the recoils. Since it was commissioned at the UND in 1997, at least 58 publications have reported data from its use and there have been hundreds of collaborators from many different countries that use this device. Currently, plans are in place at the UND to provide several upgrades to TwinSol, including a multi-cell gas production target and the possible addition of a third solenoid. Upgrades currently in progress will be discussed along with future plans.
\end{abstract}

Keywords: solenoid spectrometer, radioactive ion beams

\section{TwinSol}

TwinSol is a dual in-line superconducting solenoid system used for the production of low-energy radioactive ion beams at the University of Notre Dame (UND) Nuclear Science Laboratory. Each solenoid possesses a $30 \mathrm{~cm}$ bore, is liquid-helium cooled, and is capable of producing central fields up to 6 Tesla in strength. A primary beam bombards a production target to produce the nuclei of interest. The recoils are then collected, separated from contaminants, transported and focused onto the secondary target position. The primary beams are produced by the 10MV UND FN-Tandem accelerator which is capable of achieving beam intensities of up to $10^{12}$ particles per second with energies between 1 and $10 \mathrm{MeV} / \mathrm{u}$. For more information about TwinSol see (1) and (2).

\section{Motivation}

One of the biggest questions in science today is understanding the origin of elements in the universe. It is believed that some isotopes can only be synthesized in novae, reoccurring explosive events transpiring in binary star systems. After a nova event, the ejected envelope is opaque to gamma rays for some time. It is the observation of gamma rays from relatively long-lived isotopes, ones that decay once the envelope becomes transparent, that allows us to probe some of the properties of the nova. ${ }^{18} \mathrm{~F}$ is one such candidate. However, it is necessary to understand the reactions that produce and destroy this isotope. Two reactions of particular importance are the ${ }^{18} F(p, \alpha){ }^{15} O$ reaction which destroys it, and the ${ }^{17} \mathrm{~F}(p, \gamma){ }^{18} \mathrm{Ne}$ reaction which is

Preprint submitted to Elsevier

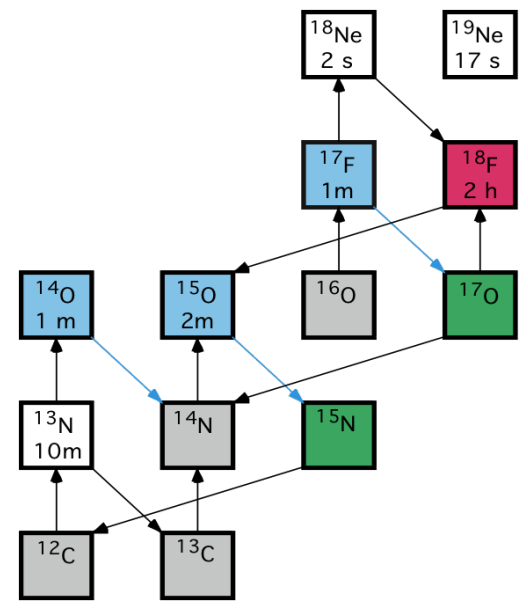

Figure 1: A representation of the primary reactions directly involved in the creation and destruction of ${ }^{18} \mathrm{~F}$.

the primary mechanism for its production, since the ${ }^{18} \mathrm{Ne}$ soon beta-decays into ${ }^{18} \mathrm{~F}$. TwinSol potentially allows us to acquire important information about both of these reactions.

\section{Multi-cell Production Target}

In order to understand the ${ }^{17} \mathrm{~F}(p, \gamma)$ reaction, we need to determine the spectroscopic strengths of certain bound states of ${ }^{18} \mathrm{Ne}$. One powerful method to do this is to use the ${ }^{17} \mathrm{~F}(d, n)$ reaction to populate those states. The outgoing neutrons would 


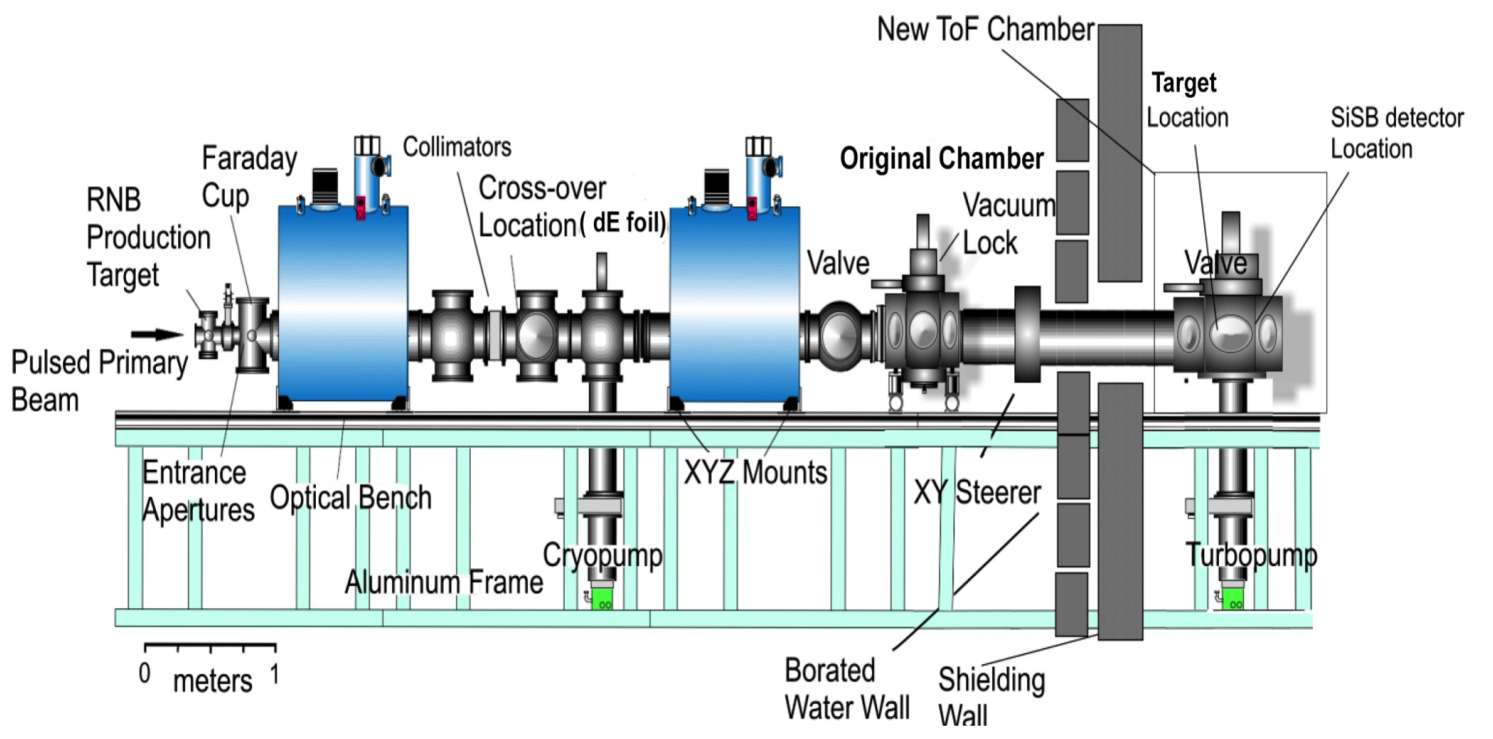

Figure 2: Schematic drawing of TwinSol.

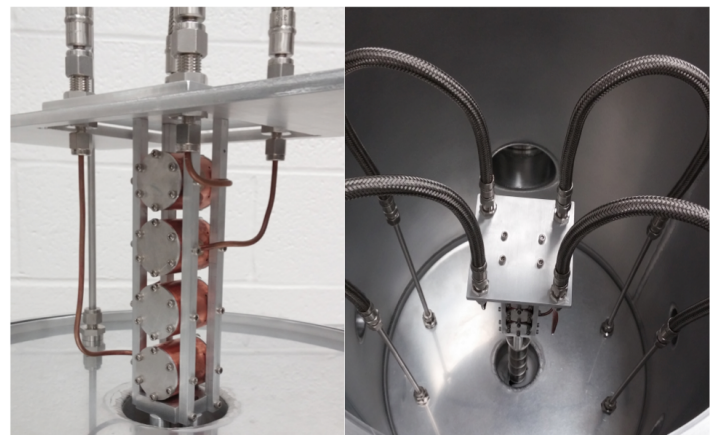

Figure 3: A picture of the new four-cell gas production target for TwinSol and the gas manifold system to supply them.

be detected by arrays of liquid and plastic scintillators, using time-of-flight to determine the neutron energies. The angular distributions would be used to extract spectroscopic information about these states. However, we first need to produce the beam. By bombarding a $D_{2}$ gas-cell production target with a primary beam of ${ }^{16} \mathrm{O}$, a secondary beam of ${ }^{17} \mathrm{~F}$ is created at intensities greater than $10^{6}$ particles per second. The resultant beam of particles would then be purified and focused by TwinSol and transported downstream where it would bombard a secondary target (a deuterated plastic in this case). Unfortunately the gas-cell windows do not last while being bombarded with so much beam power. Typically we must change production cell windows every 36 hours, which not only significantly reduces the time in which we can acquire data, but it also exposes the experimenter carrying out the replacement to an unnecessary dosage of radiation (the contact dosage is typically around 100-500 mrem/hour). In order to alleviate these problems, a multi-cell production target has been constructed.

The new production target system consists of 4 gas-cells mounted to a common mechanical feedthrough. In Figure 3 we show the four new copper cells along with the flexible, stainless-steel hoses which attach to the chamber feedthroughs. Each of these cells is approximately $2.5 \mathrm{~cm}$ long and $2 \mathrm{~cm}$ in diameter. The $5 \mu \mathrm{m}$ titanium windows can hold up to $2 \mathrm{~atm}$ of gas (typically ${ }^{3} \mathrm{He}$ or $\mathrm{D}_{2}$ ). When a window fails we simply move a new cell into position, allowing us to change production targets in a matter of minutes rather than potentially losing hours. Furthermore, these cells are mounted to a motor (controlled remotely) which can slightly oscillate, spreading the energy deposition across a wider area of the titanium window. This should prolong the life of the cells without affecting beam production. As an added bonus, the new chamber will move the target position $20 \mathrm{~cm}$ further from the entrance to the first solenoid. This reduces the angular acceptance of TwinSol, but we expect the beam energy resolution to improve by $\approx 20 \%$ with a similar reduction in beam spot size. The belief is also that this reduction in acceptance angle into TwinSol will further reduce the amount of elastically scattered primary beam that passes through the collimator without greatly reducing the yield of the beam of interest. This is largely due to the fact that the production reactions tend to be very forward peaked.

\section{SSNAP: the Solenoid Spectrometer for Nuclear Astro- Physics}

For the ${ }^{18} \mathrm{~F}+\mathrm{p}$ reaction, it is the structure of ${ }^{19} \mathrm{Ne}$ that we attempt to study and many efforts have been made in recent years to study this nucleus $(3 ; 4)$. Of particular importance are states near the proton separation energy. We wish to study these states via the ${ }^{19} \mathrm{~F}\left({ }^{3} \mathrm{He}, t\right)$ reaction. ${ }^{19} \mathrm{~F}$ is a stable nucleus so intense beams are readily available. On the other hand, ${ }^{3} \mathrm{He}$ is difficult to make into a clean and dense target. Notre Dame has the capability of producing high intensity beams of ${ }^{3} \mathrm{He}$ so 

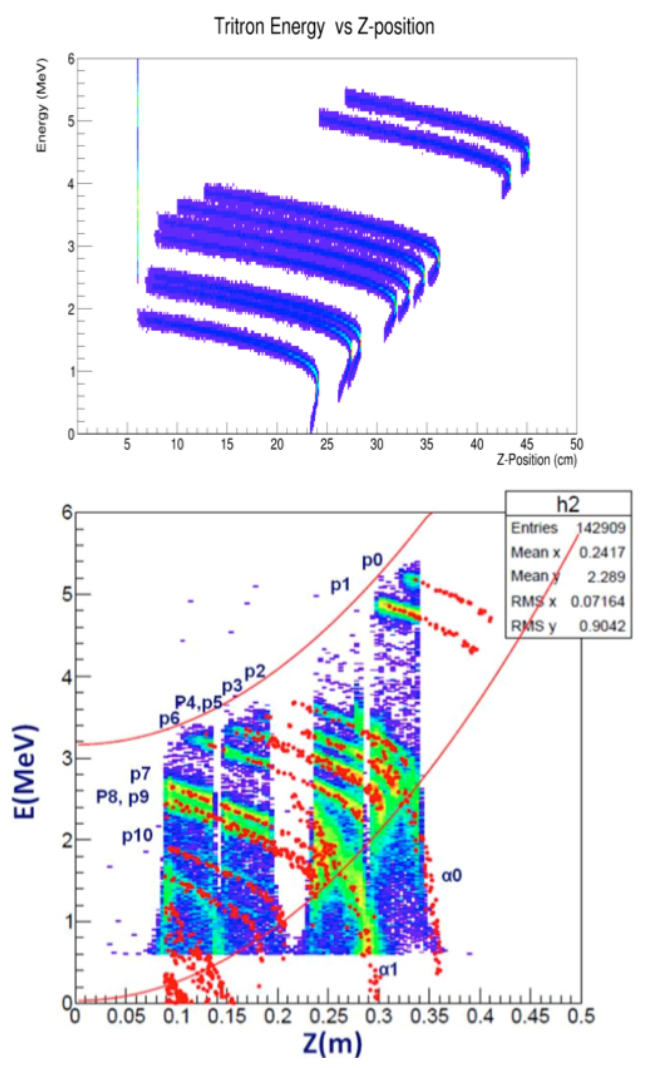

Figure 4: top) A simulation of the ${ }^{12} \mathrm{C}+{ }^{12} \mathrm{C}$ reaction (showing only the outgoing proton channels). bottom) The first test of the SSNAP concept using a beam of $10 \mathrm{MeV}{ }^{12} \mathrm{C}$ on a ${ }^{12} \mathrm{C}$ target foil. Note the different slopes for protons (over-layed with dotted black lines) and alphas (over-layed with dotted red lines). Adapted from ref. (6)

running this experiment in normal kinematics would be preferred. The energy of the out-going triton gives us some spectroscopic information about the nucleus. However, since these states are above the proton separation energy, they will emit a proton some percentage of the time. We would like to detect these low-energy protons, but we also need to separate them from the other particles being emitted, e.g. the tritons. This is non-trivial, because we are unable to use the usual technique of the $\Delta E-E$ using stacked silicon detectors and comparing the energy loss in a thin silicon detector as the particle passes through it to the total energy deposited in both layers. The protons in this case are simply too low in energy to pass through even the thinnest silicon detector available. The HELIOS spectrometer has demonstrated that particle identification can be done within a helical spectrometer based on the time-of-flight of the particle (5). This has led us to develop SSNAP, the Solenoid Spectrometer for Nuclear AstroPhysics.

SSNAP will be designed to work in conjunction with TwinSol. It will consist of a series of position sensitive silicon detectors placed just above the beam axis within the second solenoid. After the beam has bombarded a target, the ejectiles will follow a helical path back to the axis, depositing their energy into the detectors. For example, in the reaction described above, we would place the array in the second solenoid, using the first to focus the beam onto the primary target placed at the entrance to the solenoid.The radius and position of the particle are determined by their outgoing angle and energy. The conversion from $\mathrm{z}$ (position along the beam axis where the particle impacted the detector) and center of mass angle is:

$$
\theta_{c . m .}=\cos ^{-1}\left(\frac{1}{2 \pi} \frac{q e B z-2 \pi m V_{c m}}{\sqrt{2 m E_{l a b}+m^{2} V_{c m}^{2}-m V_{c m} q e B z / \pi}}\right)
$$

where $\theta_{c . m}$. is the center-of-mass angle, $q$ is the charge, $B$ is the magnetic field, $z$ is the position along the beam axis, $m$ is the mass, $E_{l a b}$ is the lab energy, and $V_{c . m}$ is the center-of-mass velocity of the detected particle. A prototype of SSNAP has been built and a test run of ${ }^{12} \mathrm{C}+{ }^{12} \mathrm{C}$ has been performed (see Figure 4). Note the separation of alphas and protons from the kinematic slopes. A simulation program has been written to help characterize the array and some results are plotted along with the data in Figure 4. Currently the designs for the array are being finalized.

\section{Future Efforts}

The multi-cell chamber has been installed at the end of June 2015. During the first week of October 2015 we will bombard the new production target to characterize the effects of oscillating the cells on both the life of the window and the beam production. We will also characterize the change in optics that moving the gas-cell has created. Currently the next generation of SSNAP is being designed and various detector formats are to be tested inside TwinSol over the next few months.

\section{Acknowledgements}

We thank the faculty, staff, and students at the University of Notre Dame for all their assistance and the many collaborators who have worked at the TwinSol facilities. The research and TwinSol-related apparatus built by University of Michigan and University of Notre Dame has been funded primarily by grants from the U.S. National Science Foundation.

Work supported in part by NSF grants PHY14-01343 and PHY-14-19765.

\section{Bibliography}

[1] F. D. Becchetti et. al. "Recent Results from the TwinSol Low-energy RIB Facility," Included in this proceedings

[2] T. Ahn, et. al. "The Prototype Active-Target Time-Projection Chamber used with TwinSol Radioactive-Ion Beams," Included in this proceedings

[3] C. E. Beer et al., Phys. Rev. C 83, 042801(R) (2011).

[4] A. M. Laird et al., Phys. Rev. Lett. 110, 032502 (2013).

[5] B. Back, "HELIOS: The HElical Orbit Spectrometer at ATLAS," ANL, pp. 8, 2010, 2010

[6] X. Fang et al., J.Phys. G: Conf. Ser. 420 (2013) 012151. 